\title{
Análisis de la frecuencia y factores de riesgo asociados a la retinopatía del prematuro en neonatos de muy bajo peso al nacer
}

\author{
María A. Rivera-Rueda ${ }^{1 *}$, Luis A. Fernández-Carrocera², Manuel B. Salgado-Valladares ${ }^{3}$, \\ Guadalupe Cordero-González², Irma A.Coronado-Zarco ${ }^{4}$ y Jorge A. Cardona-Pérez ${ }^{5}$ \\ ${ }^{1}$ Departamento de Seguimiento Pediátrico; ${ }^{2}$ Unidad de Cuidados Intensivos Neonatales; ${ }^{3}$ Coordinación de Oftalmología; ${ }^{4}$ Subdirección de \\ Neonatología; ${ }^{5}$ Dirección General. Instituto Nacional de Perinatología, Ciudad de México, México
}

\section{Resumen}

Introducción: La retinopatía del prematuro $(R O P)$ es una de las principales causas de ceguera infantil. La inmadurez y la exposición a oxígeno son algunos factores de riesgo. El objetivo de este artículo fue analizar la frecuencia y los factores de riesgo de $R O P$ en una cohorte de recién nacidos menores de 1,500 g. Métodos: Se llevó a cabo un estudio de casos (con $R O P$ ) y controles (sin ROP) de recién nacidos menores de 1,500 g. Se analizaron variables prenatales y neonatales, y para su comparación se utilizaron las pruebas estadísticas $t$ de Student, $\chi^{2}$ y U de Mann-Whitney. Resultados: Se analizaron 282 recién nacidos: 152 (53.9\%) con ROP y 130 (46.1\%) sin ROP. La mayor frecuencia se encontró en los estadios 1 y 2, con 139 pacientes (91.4\%), seguidos de los estadios 3 a 5, con 13 pacientes (8.5\%). En los pacientes con ROP, el peso al nacer fue menor (902.7 vs. $1037.9 \mathrm{~g}$; $p<0.0001$ ), así como la edad gestacional (28.2 vs. 29.6 semanas de gestación; $p<0.0001)$. Los días de ventilación (32.8 vs. 16.1; $p$ < 0.00001) y los días de oxígeno requerido durante la estancia hospitalaria (87.7 vs. 62.6; $p<0.0001$ ) fueron mayores en los pacientes con ROP. La displasia broncopulmonar, la hemorragia intraventricular $y$ la sepsis tardía fueron comorbilidades significativas para el desarrollo de ROP. Conclusiones: En este estudio, la frecuencia de ROP fue mayor que la reportada en la población mexicana, con una baja proporción de formas graves. La vigilancia estrecha del manejo de los neonatos con menor peso y menos edad gestacional es fundamental para lograr disminuir esta enfermedad.

Palabras clave: Retinopatía del prematuro. Neonatos de muy bajo peso al nacer. Neonatos prematuros.

\section{Retinopathy of prematurity, frequency and risk factors in very low birth weight infants}

\begin{abstract}
Background: Retinopathy of prematurity (ROP) is the principal cause of blindness during childhood. The objective of this study was to analyze the frequency of ROP and risk factors associated with ROP in a cohort of very low birth weight infants. Methods: A cases (ROP) and controls (no ROP) study of infants less than $1500 \mathrm{~g}$ was conducted. Perinatal and neonatal variables were analyzed. For the statistical analysis, $\chi^{2}$ test, Student's $t$-test and Mann-Whitney's U-test were used. Results: For the study, 282 neonates were included: 152 (53.9\%) with ROP and 130 (46.1\%) without ROP. The most frequent stages
\end{abstract}

Correspondencia:

*María A. Rivera-Rueda

E-mail: marivera1309@yahoo.com.mx
Disponible en internet: 23-04-2020 Bol Med Hosp Infant Mex. 2020;77(3):135-141

www.bmhim.com 1665-1146/C 2020 Hospital Infantil de México Federico Gómez. Publicado por Permanyer. Este es un artículo open access bajo la licencia CC BY-NC-ND (http://creativecommons.org/licenses/by-nc-nd/4.0/). 
observed were stage 1 and 2, with 139 (91.4\%) patients, and stages 3 to 5, with only 13 patients (8.5\%). In those neonates with ROP compared with neonates without ROP, the birth weight was less (902.7 vs. $1037.9 \mathrm{~g})$ and the difference was significant $(p<0.0001)$. Also, the difference with gestational age (28.2 vs. 29.6; $p<0.0001)$, total ventilation days (32.8 vs. 16.1; $p<0.00001)$ and total oxygen days (87.7 vs. 62.6; $p<0.0001$ ) was significant in neonates with ROP and neonates without the disease. Bronchopulmonary dysplasia, intraventricular hemorrhage and late onset sepsis were significant with patients with ROP. Conclusions: The frequency of ROP reported here is higher than the reported in Mexican population, with less cases of severe ROP. The neonatal surveillance in babies with less birth weight and gestational age is important to decrease the incidence of ROP.

Key words: Retinopathy of prematurity. Very low birth weight infant. Premature infants.

\section{Introducción}

La comprensión, el diagnóstico y el tratamiento de la retinopatía del prematuro (ROP) ha cambiado en los últimos 70 años a partir la descripción original en los Estados Unidos como fibroplasia retrolental por Stewart Clifford en $1940^{1}$ y los reportes por Terry en $1946^{2}$. La enfermedad se caracteriza por la detención inicial de la vascularización retiniana, seguida por la neovascularización. En etapas avanzadas ocurre el desprendimiento de la retina, causando pérdida visual permanente. En los países de ingreso alto, la incidencia de la ceguera asociada con la retinopatía se ha reportado menor del $10 \%$ en los prematuros de peso extremadamente bajo. En contraste, en los países de ingresos medios la incidencia es mayor del $40 \%{ }^{3}$.

La retinopatía muestra dos fases de desarrollo. La fase 1 se caracteriza por el crecimiento retardado de los vasos retinianos, que comienza desde el nacimiento hasta semanas después del nacimiento. La fase 2 se caracteriza por la neovascularización, semanas a meses después del nacimiento, y se clasifica por etapas, de acuerdo con la gravedad de la enfermedad (etapas 1 a 5), y por zona, según la localización de la ROP en la retina (zonas I a III) ${ }^{4,5}$.

Actualmente, la detección de la retinopatía que requiere tratamiento se logra mediante exámenes de la retina. En una cohorte que evaluó una guía de detección de retinopatía en Suecia se encontró el $24 \%$ de pacientes prematuros con retinopatía, de los cuales el $4.2 \%$ requirió tratamiento ${ }^{6}$. El riesgo fue mayor para la población estadounidense, donde Kennedy, et al. ${ }^{7}$ reportaron que el $14 \%$ de los lactantes analizados desarrollaron retinopatía grave y requirieron tratamiento. Kong, et al. ${ }^{8}$ informaron, a través de la red neonatal canadiense, que el $38 \%$ de los prematuros < 32 semanas de gestación (SDG) desarrollaron ROP, y de estos, el $5 \%$ requería tratamiento para la retinopatía grave.

Por otro lado, la American Academy of Pediatrics recomienda la detección de retinopatía basada en el peso al nacer $(<1,500 \mathrm{~g})$ y la edad gestacional $(<30 \text { SDG })^{9}$.

La ROP es una de las principales causas de ceguera infantil. La inmadurez y la administración excesiva de oxígeno son importantes factores de riesgo identificados para la ROP ${ }^{10}$. Algunas enfermedades se han asociado con la ROP: displasia broncopulmonar, enterocolitis necrosante, hemorragia intraventricular y sepsis $^{11-13}$. En años recientes, los investigadores han encontrado que los factores de crecimiento, como el factor de crecimiento similar a la insulina 1 y el factor de crecimiento endotelial vascular (VEGF), influyen de manera importante en el desarrollo de ROP ${ }^{4,14}$.

Por otro lado, existe evidencia experimental y clínica que demuestra que las fluctuaciones en la oxigenación se asocian con el desarrollo de ROP grave gr-18. $^{15}$.

Las terapias convencionales de la ROP se limitan al tratamiento con láser para la ablación de la retina avascular, para evitar el desprendimiento de la retina como consecuencia de la ROP ${ }^{19}$. Sin embargo, la eficacia de la terapia con láser ablativo se limita y se asocia con la destrucción de la retina, lo que causa una pérdida del campo visual clínicamente significativa.

A través del tiempo se han intentado diversos tratamientos. Por ejemplo, no es claro si el uso temprano de ácidos grasos omega-3 en forma de emulsiones lipídicas de aceite de pescado impide o disminuye la ROP. En una revisión sistemática, en la que el resultado principal fue la disminución de la incidencia de $\mathrm{ROP}^{20}$, se localizaron cuatro ensayos clínicos controlados aleatorizados y dos estudios retrospectivos en los que la administración de emulsión de aceite de pescado se asoció con una disminución en la ROP que requería terapia láser (riesgo relativo [RR]: 0.47 , intervalo de confianza al 95\% [IC 95\%]: 0.24-0.90; y RR: 0.40, IC 95\%: 0.22-0.76, respectivamente).

Otros desarrollos terapéuticos se centran en la terapia anti-VEGF (por ejemplo, la inyección intravítrea de bevacizumab, un anticuerpo anti-VEGF-A), que ha demostrado algún efecto a corto plazo en un estudio 
aleatorizado controlado. Sin embargo, el efecto a largo plazo continúa sin ser claro ${ }^{21-23}$.

Aunque la cafeína (trimetilxantina) ha sido utilizada habitualmente en las unidades de cuidados intensivos neonatales para la apnea de la prematuridad, Abdel-Hady, et al. ${ }^{24}$, en 2015, informaron que la gravedad de la ROP se redujo en el grupo de tratamiento con cafeína comparado con el grupo control. Considerando el perfil de seguridad y la capacidad de la cafeína para controlar factores angiogénicos y otras acciones vasculares, esta parece una terapia prometedora $^{25-30}$.

El objetivo del presente trabajo fue analizar la frecuencia de la ROP, además de algunos factores de riesgo asociados con su desarrollo, en una cohorte retrospectiva de neonatos de muy bajo peso al nacer $(<1,500 \mathrm{~g})$ que ingresaron a la unidad de cuidados intensivos neonatales (UCIN) del Instituto Nacional de Perinatología Isidro Espinosa de los Reyes, que cuenta con atención neonatal de tercer nivel.

\section{Métodos}

Se realizó un estudio de casos y controles anidados en una cohorte de recién nacidos con peso $<1,500 \mathrm{~g}$ ingresados a la UCIN, en el periodo comprendido de enero de 2016 a diciembre de 2018. Se incluyeron todos los recién nacidos egresados a su domicilio. Se consideraron como casos aquellos que desarrollaron ROP, y como controles los que no desarrollaron la enfermedad. Se excluyeron los neonatos sin valoración oftalmológica o con malformaciones oftálmicas. Se analizaron variables prenatales (embarazo único o múltiple, uso de esteroides prenatales y aplicación a la madre de sulfato de magnesio como neuroprotector) y neonatales (peso al nacer, edad gestacional, uso de surfactante, máxima fracción inspirada de oxígeno $\left[\mathrm{FiO}_{2}\right]$ utilizada en la sala de partos, días de ventilación, número de días de necesidad de oxígeno suplementario, comorbilidad neonatal y edad gestacional corregida de las evaluaciones oftalmológicas). Cabe mencionar que, en la institución, se sigue un control estricto de la saturación de oxígeno (del 90-95\%).

Los neonatos fueron evaluados por un médico especialista oftalmólogo, certificado, quien realizó la oftalmoscopía indirecta. La ROP se clasificó de la siguiente manera $^{31}$ :

- Estadio 1. Línea de demarcación: una fina línea blanca que separa la retina vascular de la avascular. Histológicamente: derivaciones (shunts) arteriovenosas.
- Estadio 2. Cresta borde: la línea de demarcación del estadio 1 aumenta de volumen y se extiende fuera del plano de la retina.

- Estadio 3. Crecimiento de tejido vascular (neovasos) hacia el espacio vítreo.

- Estadio 4. Desprendimiento parcial de retina: 4a) mácula aplicada, 4b) mácula desprendida.

- Estadio 5. Desprendimiento total de la retina.

Para el análisis estadístico se utilizaron medidas de tendencia central y porcentajes, t de Student, $\chi^{2}, U$ de Mann-Whitney, diferencia de medias y razón de momios con IC $95 \%$.

\section{Resultados}

La muestra estuvo constituida por 282 recién nacidos que, para fines de comparación, se dividieron en dos grupos: $152(53.9 \%)$ con retinopatía y 130 (46.1\%) sin la enfermedad. Del total de los pacientes con retinopatía, la mayor frecuencia fue para el estadio 1 , con 113 pacientes $(74.3 \%)$; en el estadio 2 se detectaron 26 pacientes (17.1\%); en el estadio 3, 12 neonatos (7.9\%); y en el estadio 5 , solo un paciente $(0.7 \%)$. De todos los pacientes con retinopatía, 22 recibieron tratamiento de ablación de retina con láser y un paciente de vitrectomía.

No se observaron diferencias significativas entre los grupos en el uso de esteroides, de sulfato de magnesio prenatal, ni en el sexo o en la calificación de Apgar < 3 al primer minuto. Se detectaron diferencias en la vía de resolución del embarazo por cesárea $(p=0.038)$. El peso al nacer fue significativamente menor en los pacientes con ROP $(902.7 \pm 178.8 \mathrm{~g}$ vs. $1037.9 \pm 213.3 \mathrm{~g} ; \mathrm{p}<$ 0.0001), así como la edad gestacional, pues los recién nacidos con ROP fueron significativamente menores en comparación con los recién nacidos sin ROP $(28.2 \pm$ 1.3 vs. $29.6 \pm 2$ SDG; $p<0.0001$ ) (Tablas 1 y 2).

$\mathrm{La} \mathrm{FiO}_{2}$ en la reanimación alcanzó valores elevados estadísticamente significativos para los pacientes con $\operatorname{ROP}(82.3 \pm 23$ vs. $70.9 \pm 27.2 \% ; p<0.0002)$, así como la ventilación ( $32.8 \pm 27.8$ vs. $16.1 \pm 20.8$ días), en comparación con los nenonatos sin ROP $(p<0.00001)$. Los prematuros con ROP requirieron más días de administración de oxígeno durante su estancia hospitalaria $(87.7 \pm 36.7$ vs. $62.6 \pm 31.2$ días; $p<0.0001)$ que los neonatos sin ROP. La displasia broncopulmonar, la hemorragia intraventricular y la sepsis tardía fueron comorbilidades significativas para el desarrollo de ROP, y no se observó diferencia significativa para conducto arterioso y enterocolitis necrosante (Tablas 1 y 2).

Al comparar los pacientes del estadio 1 con los de los estadios 2 a 5 se encontraron diferencias significativas 
Tabla 1. Características perinatales y neonatales en pacientes con retinopatía del prematuro

\begin{tabular}{|l|c|c|c|c|}
\hline & Con ROP (n= 152), $\mathbf{n}(\%)$ & Sin ROP (n= 130), n (\%) & RM (IC 95\%) & $\mathbf{p}^{*}$ \\
\hline Esteroides prenatales & $130(85.5)$ & $108(83.1)$ & $1.2(0.63,2.29)$ & 0.57 \\
\hline Sulfato de magnesio & $107(70.4)$ & $86(66.2)$ & $1.21(0.73,2.01)$ & 0.44 \\
\hline Cesárea & $116(76.3)$ & $112(86.2)$ & $0.52(0.28,0.96)$ & 0.038 \\
\hline Sexo masculino & $87(57.2)$ & $70(53.8)$ & $1.14(0.71,1.83)$ & 0.57 \\
\hline Sexo femenino & $65(42.8)$ & $60(46.2)$ & $0.87(0.54,1.40)$ & 0.57 \\
\hline Apgar <3 a 1 minuto & $50(32.9)$ & $44(33.9)$ & $0.95(0.58,1.57)$ & 0.86 \\
\hline Displasia broncopulmonar & $149(98)$ & $117(90)$ & $5.51(1.53,19.8)$ & 0.0088 \\
\hline Hemorragia intraventricular & $70(46)$ & $34(26.2)$ & $2.41(1.45,3.99)$ & 0.0006 \\
\hline Sepsis tardía & $102(67.1)$ & $54(41.5)$ & $2.76(1.7,4.47)$ & $<0.0001$ \\
\hline Conducto arterioso & $94(61.8)$ & $66(50.8)$ & $1.57(0.98,2.53)$ & 0.062 \\
\hline Enterocolitis necrosante & $45(29.6)$ & $27(20.8)$ & $1.6(0.93,2.78)$ & 0.09 \\
\hline
\end{tabular}

*Obtenida utilizando la prueba de $\chi^{2}$.

IC: intervalo de confianza; RM: razón de momios; ROP: retinopatía del prematuro.

Tabla 2. Características neonatales en pacientes con retinopatía del prematuro

\begin{tabular}{|l|c|c|c|c|}
\hline & Con ROP $(\mathbf{n}=\mathbf{1 5 2}), \mathbf{X} \pm \mathbf{D E}$ & Sin ROP $(\mathbf{n}=\mathbf{1 3 0}), \mathbf{X} \pm \mathbf{D E}$ & $\mathbf{D M}(\mathbf{C C} \mathbf{9 5} \%)$ & $\mathbf{p}^{*}$ \\
\hline Peso al nacer (g) & $902.7 \pm 178.8$ & $1037.9 \pm 213.3$ & $135.2(89.24,181.2)$ & $<0.0001$ \\
\hline Edad gestacional (semanas) & $28.2 \pm 1.3$ & $29.6 \pm 2.0$ & $1.4(1.01,1.79)$ & $<0.0001$ \\
\hline FiO ${ }_{2}$ máxima en UT0 (\%) & $82.3 \pm 23$ & $70.9 \pm 27.2$ & $-11.4(-17.28,-5.5)$ & $<0.0002$ \\
\hline Ventilación (días) & $32.8 \pm 27.8$ & $16.1 \pm 20.8$ & - & $<0.00001$ \\
\hline CPAP (días) & $15.5 \pm 15.1$ & $16.8 \pm 14.3$ & - & 0.238 \\
\hline Oxígeno (días) & $87.7 \pm 36.7$ & $62.6 \pm 31.2$ & $-25.1(-33.16,-17.04)$ & $<0.0001$ \\
\hline
\end{tabular}

*Obtenida utilizando las pruebas $t$ de Student y $U$ de Mann-Whitney.

CPAP: presión positiva continua en la vía aérea; DM: diferencia de medias; IC: intervalo de confianza; FiO 2 : máxima fracción inspirada de oxígeno; ROP: retinopatía del prematuro; UTQ: unidad tocoquirúrgica; $\mathrm{X} \pm \mathrm{DE}$ : promedio \pm desviación estándar.

en cuanto al peso (mayor en el estadio 1), la edad gestacional (mayor en el estadio 1), la $\mathrm{FiO}_{2}$ máxima en la unidad tocoquirúrgica (menor en el estadio 1) y el número de días de ventilación, así como en la exposición al oxígeno (menor en el estadio 1) (Tabla 3).

En la Tabla 4 se muestra la edad gestacional corregida a la cual fueron valorados por primera vez los neonatos participantes del estudio, dependiendo de su edad gestacional al nacer.

\section{Discusión}

La ROP es una de las principales causas de ceguera infantil. La inmadurez y la administración excesiva de oxígeno constituyen el primer factor de riesgo identificado ${ }^{32}$.

En este estudio, la retinopatía en general se presentó en el $53.9 \%$ de los casos, lo cual concuerda con los resultados de otros países con ingresos medios ${ }^{33}$. Sin embargo, los resultados de diversos países de la Red Internacional para la Evaluación de los Resultados de los recién nacidos indican tasas del 25.2 al 91\% para cualquier ROP, en un total de 48,087 lactantes de 24-27 SDG incluidos en el análisis ${ }^{34}$. Con excepción de Japón, se ha visto que la diversidad étnica también puede contribuir a la variación observada. Se ha informado de un menor riesgo de ROP en neonatos afroamericanos en comparación con los de raza 
Tabla 3. Características neonatales de los pacientes con retinopatía del prematuro según el estadio

\begin{tabular}{|l|c|c|c|c|}
\hline & Estadio $\mathbf{1}(\mathbf{n}=\mathbf{1 1 3}), \mathbf{X} \pm \mathbf{D E}$ & Estadio $\mathbf{2 - 5}(\mathbf{n}=\mathbf{3 9}), \mathbf{X} \pm \mathbf{D E}$ & $\mathbf{D M}(\mathbf{I C} \mathbf{9 5} \%)$ & $\mathbf{p}^{*}$ \\
\hline Peso al nacer (g) & $923.5 \pm 187.5$ & $842.6 \pm 135.6$ & $-80.9(-145.4,-16.38)$ & 0.0143 \\
\hline Edad gestacional (semanas) & $28.4 \pm 1.3$ & $27.5 \pm 1.1$ & $-0.9(-1.36,-0.44)$ & 0.0002 \\
\hline FiO ${ }_{2}$ máxima en UT0 & $81.1 \pm 23.9$ & $85.9 \pm 20.1$ & $4.8(-3.64,13.24)$ & 0.263 \\
\hline Ventilación (días) & $30.1 \pm 26.3$ & $40.8 \pm 30.6$ & - & 0.0394 \\
\hline CPAP (días) & $14.4 \pm 15.1$ & $18.5 \pm 15.1$ & - & 0.062 \\
\hline Oxígeno (días) & $83.9 \pm 35.4$ & $98.7 \pm 38.7$ & $14.8(1.49,28.10)$ & 0.029 \\
\hline
\end{tabular}

*Obtenida utilizando las pruebas t de Student y U de Mann-Whitney.

CPAP: presión positiva continua en la vía aérea; DM: diferencia de medias; IC: intervalo de confianza; $\mathrm{FiO}_{2}$ : máxima fracción inspirada de oxígeno; ROP: retinopatía del prematuro; UTQ: unidad tocoquirúrgica; $\mathrm{X} \pm \mathrm{DE}$ : promedio \pm desviación estándar.

Tabla 4. Edad gestacional corregida en la primera evaluación oftalmológica

\begin{tabular}{|l|c|c|}
\hline $\begin{array}{l}\text { Edad gestacional } \\
\text { al nacer (semanas) }\end{array}$ & $\begin{array}{c}\text { Edad gestacional corregida de } \\
\text { la primera valoración (semanas) }\end{array}$ & $\mathbf{n = 2 8 2}$ \\
\hline$<27$ & $30.8 \pm 1.27$ & 27 \\
\hline 27 & $32.9 \pm 2.04$ & 36 \\
\hline 28 & $33.2 \pm 1.67$ & 86 \\
\hline 29 & $34.02 \pm 1.48$ & 51 \\
\hline 30 & $34.6 \pm 1.3$ & 44 \\
\hline 31 & $36.1 \pm 1.86$ & 19 \\
\hline 32 & $38.1 \pm 3.5$ & 8 \\
\hline$\geq 33$ & $39.2 \pm 2.13$ & 11 \\
\hline
\end{tabular}

blanca, mientras que los hispanos y los asiáticos de la India mostraron un mayor riesgo ${ }^{34-37}$.

En una cohorte mexicana ${ }^{38}$ estudiada en la ciudad de Guadalajara, Jalisco, de 85 prematuros < 34 SDG $\mathrm{y} \leq 1750 \mathrm{~g}$, el $39 \%$ presentó cualquier tipo de retinopatía; cifra menor que la observada en el presente estudio. Se debe considerar que la población de este estudio es de menor peso y menor edad gestacional. Los autores detectaron el $12 \%$ de formas graves, tomando en cuenta el total de la población estudiada $(n=85)$. Si en los resultados presentes se considera el total de la muestra, el porcentaje disminuye al $4.6 \%$. El $8.6 \%$ de los casos graves reportados en este informe se relaciona con los casos de retinopatía. En cambio, en el estudio de Guadalajara, el porcentaje aumenta al $30.3 \%$ si se analizan únicamente los casos de retinopatía. En general, diversos resultados refieren una media del $50 \%$ y un estadio 3 del 1.5 al 14\% para todas las ROP $7,8,32-34,38-40$.
Keraan, et al..$^{33}$ consideran el nacimiento por cesárea como un posible factor de disminución de riesgo para desarrollar ROP en niños prematuros $(p=0.007)$. En el presente trabajo, la cesárea fue un factor significativo. Sin embargo, esta observación se debe tomar con cierta reserva, ya que no existe suficiente evidencia, además de que intervienen una serie de factores para indicar una cesárea en pacientes prematuros.

El peso al nacer y la edad gestacional son factores de riesgo ya conocidos, como se pudo demostrar en este trabajo, en el que los pacientes de muy bajo peso y corta edad gestacional fueron los que presentaron ROP con una diferencia significativa; esta relación también ha sido demostrada por otros autores ${ }^{7,33,34,38}$.

Las altas concentraciones de oxígeno han sido ampliamente documentadas en la literatura como factor de riesgo para ROP. En este reporte, la concentración elevada de oxígeno, en la reanimación y los días de la ventilación mecánica convencional, no convencional y no invasiva, fue significativa para los neonatos que cursaron con ROP. Colaizy, et al. ${ }^{32}$ compararon dos cohortes, una sin protocolo de oxígeno (20022007) y otra con oxígeno controlado (2008-2012), con el objetivo de observar la progresión del estadio 2 al estadio 3. La progresión para el primer periodo fue del $44 \%$, mientras que para el segundo fue del $23 \%$, lo que resultó una disminución significativa en recién nacidos prematuros sin que se observara un aumento de la morbilidad pulmonar. Zepeda-Romero, et al. ${ }^{38}$, en el Hospital Civil de Guadalajara, México, concluyeron que, sin control, el suplemento de oxígeno es el principal factor de riesgo de la retinopatía grave del prematuro. En su estudio, después del control de oxígeno la frecuencia del tratamiento de ROP se redujo drásticamente. 
La comorbilidad es consecuencia de la prematuridad, y algunas enfermedades pueden asociarse con algún componente que incida de manera indirecta en la retinopatía. La hemorragia intraventricular es frecuente en los prematuros, sobre todo en los de muy bajo peso y corta edad gestacional; en la presente muestra, la significación fue de $p=0.006$ para los pacientes con retinopatía. La sepsis tardía fue una patología altamente significativa $(p<0.0001)$, como se ha documentado en diversas publicaciones, al igual que la displasia broncopulmonar. La enterocolitis necrosante no resultó significativa, a diferencia de varios reportes $4,20,33,39,41$.

El seguimiento de los neonatos de muy bajo peso al nacer para la detección oportuna de la retinopatía se encuentra establecido en México en las Guías de Práctica Clínica de retinopatía del prematuro. Se dispone que, de acuerdo con la edad gestacional al nacer, la primera revisión debe realizarse entre las 4 y 5 semanas posteriores al nacimiento ${ }^{31}$. En este estudio se observó que los neonatos de 27 y 28 SDG mostraron, en promedio, un retraso de 1 semana en su evaluación, por lo que habrá que apegarse más a las recomendaciones para una detección oportuna.

En la población estudiada, la retinopatía en general se presentó en el $53.9 \%$, lo cual concuerda con los resultados en otros países, aunque es mayor que lo reportado en la población mexicana, con una baja frecuencia de formas graves. El bajo peso, la edad gestacional y las concentraciones altas de oxígeno son factores de riesgo importantes para la enfermedad. La sepsis tardía fue la única comorbilidad que se presentó de forma significativa.

Una mejor atención, incluyendo la administración protocolizada de oxígeno y el seguimiento para prematuros, favorecería la reducción del número de prematuros afectados con ROP. Se requiere una acción urgente para mejorar el conocimiento de la ROP entre todos los profesionales sanitarios implicados en la atención de los recién nacidos prematuros, junto con los compromisos para mejorar la atención neonatal, así como desarrollar y poner en práctica protocolos y directrices para la prevención, la detección y el tratamiento de la ROP.

\section{Responsabilidades éticas}

Protección de personas y animales. Los autores declaran que para esta investigación no se han realizado experimentos en seres humanos ni en animales.
Confidencialidad de los datos. Los autores declaran que han seguido los protocolos de su centro de trabajo sobre la publicación de datos de pacientes.

Derecho a la privacidad y consentimiento informado. Los autores han obtenido el consentimiento informado de los pacientes y sujetos referidos en el artículo. Este documento obra en poder del autor de correspondencia.

\section{Conflicto de intereses}

Los autores declaran no tener ningún conflicto de intereses.

\section{Financiamiento}

\section{Ninguno.}

\section{Bibliografía}

1. Robertson AF. Reflections on errors in neonatology: I. The "hands-off" years, 1920 to 1950. J Perinatol. 2003;23:48-55.

2. Terry TL. Retrolental fibroplasia. J Pediatr. 1946;29:770-3.

3. Rivera JC, Holm M, Austeng D, Morken TS, Zhou TE, Beaudry-Richard A, et al. Retinopathy of prematurity: inflammation, choroidal degeneration, and novel promising therapeutic strategies. I Neuroinflammation. 2017;14:165-87.

4. Lundgren $P$, Lundberg L, Hellgren G, Holmström G, Hård AL, Smith LE, et al. Aggressive posterior retinopathy of prematurity is associated with multiple infectious episodes and thrombocytopenia. Neonatology. 2017;111:79-85.

5. International Committee for the Classification of Retinopathy of Prematurity. The International Classification of Retinopathy of Prematurity revisited. Arch Ophthalmol. 2005;123:991-9.

6. Holmström G, Hellström A, Jakobsson P, Lundgren $P$, Tornqvist $K$, Wallin A. Evaluation of new guidelines for ROP screening in Sweden using SWEDROP - a national quality register. Acta Ophthalmol. 2015; 93:265-8.

7. Kennedy KA, Wrage LA, Higgins RD, Finer NN, Carlo WA, Walsh MC, et al. Evaluating retinopathy of prematurity screening guidelines for 24- to 27-week gestational age infants. J Perinatol. 2014;34:311-8.

8. Kong L, Fry M, Al-Samarraie M, Gilbert C, Steinkuller PG. An update on progress and the changing epidemiology of causes of childhood blindness worldwide. J AAPOS. 2012;16:501-7.

9. Fierson WM, American Academy of Pediatrics Section on Ophtalmology, American Academy of Ophthalmology, American Association of Pediatrics Ophthalmology and Strabismus. Screening examination of premature infants for retinopathy of prematurity. Pediatrics. 2006;117:572-6.

10. Ashton N, Ward B, Serpell G. Effect of oxygen on developing retinal vessels with particular reference to the problem of retrolental fibroplasia. Br J Ophthalmol. 1954;38:397-432.

11. Holmström G, Broberger U, Thomassen P. Neonatal risk factors for retinopathy of prematurity a population-based study. Acta Ophthalmol Scand. 1998;76:204-7.

12. Arroe M, Peitersen B. Retinopathy of prematurity: review of a seven-year period in a Danish neonatal intensive care unit. Acta Paediatr. 1994;83:501-5.

13. Klinger G, Levy I, Sirota L, Boyko V, Lerner-Geva L, Reichman B, et al. Outcome of early-onset sepsis in a national cohort of very low birth weight infants. Pediatrics. 2010;125:e736-40.

14. Lee JE. Low IGF-1 suppresses VEGF-survival signaling in retinal endothelial cells: direct correlation with clinical retinopathy of prematurity, by $A$. Hellström, C. Perruzzi, M. Ju, E. Engström, A Hård, J. Liu, K. Albertson-Wikland, B. Carlsson, A. Niklasson, L. Sjödell, D. LeRoith, D. Senger, and L. Smith. PNAS 98:5804-8, 2001. Surv Ophthalmol. 2003;48:234-5.

15. Cunningham S, Fleck BW, Elton RA, Mclntosh N. Transcutaneous oxygen levels in retinopathy of prematurity. Lancet. 1995;346:1464-5.

16. Penn JS, Tolman BL, Lowery LA. Variable oxygen exposure causes preretinal neovascularisation in the newborn rat. Invest Ophthalmol Vis Sci. 1993;34:576-85. 
17. York JR, Landers S, Kirby RS, Arbogast PG, Penn JS. Arterial oxygen fluctuation and retinopathy of prematurity in very-low-birth-weight infants. J Perinatol. 2004;24:82-7.

18. Hartnett ME. Advances in understanding and management of retinopathy of prematurity. Surv Ophthalmology. 2017;62:257-76.

19. Clark, D, Mandal K. Treatment of retinopathy of prematurity. Early Hum Dev. 2008; 84:95-9.

20. Vayalthrikkovil S, Bashir RA, Rabi Y, Amin H, Spence JM, Robertson HE, et al. Parenteral fish-oil lipid emulsions in the prevention of severe retinopathy of prematurity: a systematic review and meta-analysis. Am J Perinatol. 2017:34:705-15.

21. Mintz-Hittner HA, Kennedy KA, Chuang AZ, BEAT-ROP Cooperative Group. Efficacy of intravitreal bevacizumab for stage 3+ retinopathy of prematurity. N Engl J Med. 2011;364:603-15.

22. Tokunaga CC, Mitton KP, Dailey W, Massoll C, Roumayah K, Guzman E, et al. Effects of anti-VEGF treatment on the recovery of the developing retina following oxygen-induced retinopathy. Invest Ophthalmol Vis Sci. 2014;55:1884-92.

23. Hu J, Blair MP, Shapiro MJ, Lichtenstein SJ, Galasso JM, Kapur R Reactivation of retinopathy of prematurity after bevacizumab injection. Arch Ophthalmol. 2012;130:1000-6.

24. Abdel-Hady $\mathrm{H}$, Nasef $\mathrm{N}$, Shabaan $\mathrm{AE}$, Nour I. Caffeine therapy in preterm infants. World J Clin Pediatr. 2015;4:81-93.

25. Schmidt B, Roberts RS, Davis P, Doyle LW, Barrington KJ, Ohlsson A, et al. Long-term effects of caffeine therapy for apnea of prematurity. N Engl J Med. 2007;357,1893:902.

26. Fredholm BB, Bättig K, Holmén J, Nehlig A, Zvartau EE. Actions of caffeine in the brain with special reference to factors that contribute to its widespread use. Pharmacol Rev. 1999;51:83-133.

27. Ryzhov S, McCaleb JL, Goldstein AE, Biaggioni I, Feoktistov I. Role of adenosine receptors in the regulation of angiogenic factors and neovascularization in hypoxia. J Pharmacol Exp Ther. 2007;320:565-72.

28. Hsu SJ, Lee FY, Wang SS, Hsin IF, Lin TY, Huang HC, et al. Caffeine ameliorates hemodynamic derangements and portosystemic collaterals in cirrhotic rats. Hepatology. 2015:61:1672-84.

29. Li H, Jin SY, Son HJ, Seo, JH, Jeong GB. Caffeine-induced endothelial cell death and the inhibition of angiogenesis. Anat Cell Biol. 2013;46:57-67.
30. Echeverri D, Montes FR, Cabrera M, Galán A, Prieto A. Caffeine's vascular mechanisms of action. Int J Vasc Med. 2010;2010:834060-9.

31. Hernández GC, Villavicencio TA, Méndez MN, Garza RC, Espinosa LL, Millán GK. Detección, diagnóstico y tratamiento de retinopatía del prematuro en el segundo y tercer nivel de atención. Evidencias y recomendaciones. México: Secretaría de Salud; 2015.

32. Colaizy TT, Longmuir S, Gertsch K, Abramoff MD, Klein JM. Use of a supplemental oxygen protocol to suppress progression of retinopathy of prematurity. Invest Ophthalmol Vis Sci. 2017;58:887-91.

33. Keraan Q, Tinley C, Horn A, Pollock T, Steffen J, Joolay Y. Retinopathy of prematurity in a cohort of neonates at Groote Schuur Hospital, Cape Town, South Africa. S Afr Med J. 2016;107:64-69.

34. Darlow BA, Lui K, Kusuda S, Reichman B, Håkansson S, Bassler D, et al. International variations and trends in the treatment for retinopathy of prematurity. Br J Ophthalmol. 2017;101:1399-404.

35. Saunders RA, Donahue ML, Christmann LM, Pakalnis AV, Tung B, Hardy RJ, et al. Racial variation in retinopathy of prematurity. The cryotherapy for retinopathy of prematurity cooperative group. Arch Ophthalmol. 1997:115:604-8.

36. Ludwig CA, Chen TA, Hernandez-Boussard T, Moshfeghi AA, Moshfeghi DM. The epidemiology of retinopathy of prematurity in the United States. Ophthalmic Surg Lasers Imaging Retina. 2017;48:553-62.

37. Husain SM, Sinha AK, Bunce C, Arora P, Lopez W, Mun KS, et al. Relationships between maternal ethnicity, gestational age, birth weight, weight gain, and severe retinopathy of prematurity. J Pediatr. 2013;163:67-72.

38. Zepeda-Romero LC, Lundgren P, Gutierrez-Padilla JA, Gomez-Ruiz LM, Quiles-Corona M, Orozco-Monroy JV, et al. Oxygen monitoring reduces the risk for retinopathy of prematurity in a Mexican population. Neonatology. 2016:110:135-40.

39. Ahn YJ, Hong KE, Yum HR, Lee JH, Kim KS, Youn YA, et al. Characteristic clinical features associated with aggressive posterior retinopathy of prematurity. Eye (Lond). 2017;31:924-30.

40. Maroufizadeh S, Almasi-Hashiani A, Omani Samani R, Sepidarkish M. Prevalence of retinopathy of prematurity in Iran: a systematic review and meta-analysis. Int J Ophthalmol. 2017;10:1273-9.

41. Leng $Y$, Huang $W$, Ren $G$, Cai $C$, Tan $Q$, Liang $Y$, et al. The treatment and risk factors of retinopathy of prematurity in neonatal intensive care units. BMC Ophtalmol. 2019;18:301-8. 